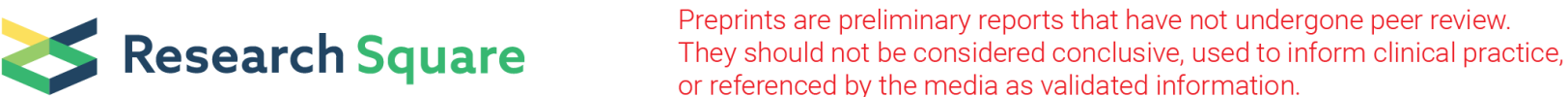

\section{Prioritizing delivery of cancer treatment during a COVID19 lockdown - the experience of a clinical oncology service in India}

Indranil Mallick ( $\square$ indranil.mallick@tmckolkata.com )

Tata Medical Center https://orcid.org/0000-0002-5567-9204

Santam Chakraborty

Tata Medical Center https://orcid.org/0000-0003-3580-5979

Shweta Baral

Tata Medical Center

Saheli Saha

Tata Medical Center

Vishnu H Lal

Tata Medical Center

Rohit Sasidharan

Tata Medical Center

Samarth Chhatbar

Tata Medical Center

Subecha Bhushal

Tata Medical Center

Love Goyal

Tata Medical Center

Shaurav Maulik

Tata Medical Center

Vezokhoto Phesao

Tata Medical Center

Siddharth Arora

Tata Medical Center

Anurupa Mahata

Tata Medical Center

Sriram Prasath

Tata Medical Center

B Arun

Tata Medical Center

Samar Mandal 
Tata Medical Center

Tapesh Bhattacharyya

Tata Medical Center

Moses Arunsingh

Tata Medical Center

Rimpa Basu Achari

Tata Medical Center

Sanjoy Chatterjee

Tata Medical Center

\section{Research Article}

Keywords: COVID-19, Coronavirus, Lockdown, Radiotherapy, Chemotherapy, Cancer

Posted Date: July 7th, 2020

DOI: https://doi.org/10.21203/rs.3.rs-38180/v1

License: (c) (i) This work is licensed under a Creative Commons Attribution 4.0 International License. Read Full License

Version of Record: A version of this preprint was published at JCO Global Oncology on July 7th, 2020. See the published version at https://doi.org/10.1200/G0.20.00433. 


\section{Abstract}

INTRODUCTION:

A COVID19 lockdown in India posed significant challenges to the continuation of radiotherapy (RT) and systemic therapy services. While several COVID19 service guidelines have been promulgated, implementation data is yet unavailable. We performed a comprehensive audit of the implementation of services in a clinical oncology department.

METHODS AND MATERIALS:

A departmental protocol of priority-based treatment guidance was developed and a departmental staff rotation policy was implemented. Data was collected for the period of lockdown on outpatient visits, starting, and delivery of RT and systemic therapy. Adherence to protocol was audited, and factors affecting change from pre-COVID standards analyzed by multivariate logistic regression.

\section{RESULTS:}

Outpatient consults dropped by $58 \%$. Planned RT starts were implemented in $90 \%, 100 \%, 92 \%, 90 \%$ and $75 \%$ of priority level $1-5$ patients. While $17 \%$ had a deferred start, the median time to start of adjuvant RT and overall treatment times were maintained. Concurrent chemotherapy was administered in $89 \%$ of those eligible. Systemic therapy was administered to $84.5 \%$ of planned patients with $33 \%$ and $57 \%$ of curative and palliative patients receiving modified or deferred cycles. The patient's inability to come was the commonest reason for RT or ST deviation. Factors independently associated with a change from preCOVID practice was priority level allocation for RT and age and palliative intent for systemic therapy.

CONCLUSIONS:

Despite significant access limitations a planned priority-based system of delivery of treatment could be implemented.

\section{Introduction}

In the wake of the COVID19 pandemic, India went into a strict lockdown on March 24, 2020. This included the abrupt cessation of all types of public and private transport except for defined essential services 1 . The sudden lockdown resulted in oncology services in India facing a crisis of decision-making and delivery of care.

From March 2020, guidelines on cancer treatment, and risk-stratified care had started emerging2. These generally suggested modification or deferment of treatment if considered safe. Although oncology services around the country started adopting one or more of the recommendations $3-5$, the available literature is limited to consensus guidelines and surveys primarily based on western healthcare 
infrastructure. Implementation of a planned approach from a system that does not have structured state funding for travel and treatment has not yet been audited or reported.

We put in place a detailed protocol to prioritize care pathways using available evidence, biological rationale and published consensus statements (Appendix I). We present here an audit of our services from March 24 through May 16, 2020. The focus of this audit was the implementation of treatment delivery amongst our patients.

\section{Methods}

\section{Departmental triaging and treatment protocols}

The departmental policy (Appendix I) was based on the treatment priorities influenced by treatment intent and disease biology. Prioritization for radiotherapy (RT) was primarily based on the recommendations of the NHS UK and divided cancer cases into five levels2. For patients on systemic therapy, we used a modified priority system based on the curative or palliative intent of therapy. We maintained a prospective database of all cases whose treatment was deferred during this period.

\section{Data sources}

We obtained patient visit data between January 1st - May 16th for the years 2019 and 2020 from the electronic hospital information system (HIS). The HIS and oncology information system (ARIA, Varian Medical System, Palo Alto, USA) was queried to obtain information on patient characteristics and treatment delivery patterns between March 24th - May 16th, 2020. Study data were collected and managed using REDCap electronic data capture tools6, 7.

Statistical analysis

R8 and Python 3 were used for statistical analysis. We used the Chi-square test and the Kruskal Wallis test was used for statistical testing of differences in frequencies and continuous variables respectively.

Multivariate modeling was used to identify the factors predicting deviation of radiotherapy and chemotherapy from pre-COVID protocols (Appendix II). Multivariate analysis was done using logistic regression, where the presence of any deviation was considered as the independent variable. Model predictors were added linearly and no interactions were assumed. Odds ratios, $95 \%$ confidence intervals, and $p$ values are presented. A $p$-value of $<0.05$ is considered statistically significant.

\section{Results}

\section{Outpatient visits}

Within the period of Jan 1- May 16, 5291, and 5090 patients had outpatient visits in 2019 and 2020 respectively. There were 12325 outpatient consults between Jan 1 to May 16, 2020, compared to 13140 
in the same period in 2019. While there were 1983 (25\%) excess outpatient visits in the first 12 weeks of 2020 as compared to 2019 , there was a sharp drop in patient visits induced by the lockdown in the 13th week. The average weekly follow up visits in the four most common site-groups of breast, lung, head and neck, and prostate cancers dropped by $65 \%, 49 \%, 50 \%$, and $76 \%$ respectively.

\section{Radiation therapy}

During the period of lockdown, there were 305 patients who were planned for starting RT. Of these, 262 were able to start on treatment between the period of 24th March to 31st May 2020 (Table 1). Breast (27\%), head and neck (23\%), and lung (17\%) were the commonest sites(Appendix II, Table 1). Of the 145 patients in priority levels 1,2 , and $3,132(91.0 \%)$ could begin their treatment during the lockdown.

In 125 (47.7\%) patients, either adjuvant RT after surgery or definitive RT after induction chemotherapy was delivered. Of these, 54 patients had priority level 1, while 69 had priority level 5 . The median time to start RT was 40 days (range: 14 - 69 days) after surgery or the last cycle of chemotherapy. A delay of 6 weeks in starting RT was observed in 17 (31.5\%) patients. In all but one of these patients, the delay beyond 6 weeks was due to restrictions in patient travel, finances, or due to delayed attendance in our hospital after a surgery done in another hospital. Among priority 5 patients undergoing adjuvant radiotherapy, the median gap between $\mathrm{RT}$ and the last cycle of chemotherapy or surgery was 53 days (range: 16 - 110 days).

The median RT plan turnaround time (TAT) was 7 days. Among priority level 1 patients, plan TAT exceeding 14 days was observed in $3(2.6 \%)$ patients. Plan TAT did not exceed 14 days in any of the priority 1 patients on adjuvant or post-induction RT. All patients in priority level 2 started on the same day of planning.

Concurrent chemotherapy was indicated in 65 (32.0\%) of the 203 patients. Amongst these 58 (89.2\%) patients were started on chemotherapy. Chemotherapy could not be delivered per protocol in 26 patients $(27.1 \%)$ due to treatment-related toxicities. Of these, 7 patients had chemotherapy stopped early, 1 had a dose reduction while 9 had missed one or more cycles of chemotherapy.

\section{Delivery of RT}

429 patients underwent RT during lockdown (262 new and 167 ongoing) (Table 2, Appendix II). By deferring starts of priority level 5 patients, on-treatment numbers reduced to an average of 129 per day during the period of lockdown from 172 earlier (Fig 1, Appendix II). A total of 8 patients had breaks, and six patients could not complete their planned treatment.

Of the six, two were unable to come for further therapy, while the remaining progressed or died during treatment (unrelated to COVID). Overall treatment time was prolonged by more than 3 days in 14 patients (5 priority level 1 ). 


\section{Downtime}

We faced a great challenge with downtime, with one of the 4 treatment units down for technical reasons on 24 days of the 59 working days (inclusive of Saturdays). However timely shifting of patients to alternative units was done which is reflected in the overall treatment time.

\section{Brachytherapy}

25 patients with gynecological cancers were planned for brachytherapy. Of these, brachytherapy could be delivered in 17 patients (11: cervical cancer, 5: endometrial cancer, 1: vault recurrence). A scheduled brachytherapy source exchange had to be deferred during the lockdown as a result of which 8 patients (4 cervical cancer and endometrial cancers each) were referred outside for brachytherapy after May 1, 2020.

In the 17 patients who received brachytherapy, treatment was completed in 15 patients. 2 patients with endometrial cancers were unable to come for the last fraction of vaginal brachytherapy. The total duration of treatment for patients who underwent treatment during this period was less than 56 days for all except 1 patient.

\section{Factors affecting deviation from pre-COVID usual radiotherapy practice}

Factors affecting deviation from practice are shown in Appendix II tables 3 and 4. Figure 2 (panel A) shows that the only factor which was independently associated with deviation from pre-COVID protocol was the priority level. Compared to priority level 1, priority level 5 had an odds ratio of 4.02 (1.53 to 10.63, $p=0.005)$ for a change or deviation. Priority level 2 had less deferment - odds ratio of 0.08 (0.01 to 0.71 , $p=0.02$ ). This was in keeping with our protocol during the lockdown.

\section{Systemic therapy}

\section{Starting planned systemic therapy}

ST was indicated in 395 patients, of whom, 61 patients could not start on treatment during the lockdown period. The most common reasons for this were patient default ( $n=31,50.8 \%)$, patient unfitness to receive systemic therapy $(n=13,21.3 \%)$ and COVID 19 related concerns $(n=8,13.1 \%)$.

Compliance in those who received chemotherapy

After excluding patients for targeted therapy, chemotherapy was delivered in 219 patients. 95 patients (43.4\%) received curative-intent treatment. Combination chemotherapy was utilized in 126 patients (57.5\%).

Table 2 shows the implementation of chemotherapy in these patients. About one-third of curative intent chemotherapy and close to $60 \%$ of patients on palliative chemotherapy had some form of deferral from planned dates. The median duration of delay was longer in palliative patients (28 days vs 7.5 days, $p=$ 
0.002). Deferrals in curative patients were equally related to disease or toxicity related causes, inability to attend due to lockdown and physician recommendations. Deferrals in patients on palliative systemic therapy were more commonly due to physician recommendation (56.4\%). In a smaller proportion of patients, there was a change of chemotherapy schedule, mainly related to reduced intensity. In only 26 (12\%) patients chemotherapy was stopped completely, and 18 of these patients were on palliative treatment. Toxicity-related stoppage or deferral was uncommon.

\section{Factors affecting deviation from pre-COVID chemotherapy practice}

Factors affecting deviation from usual practice are shown in Appendix II tables 5 and 6 . Figure 2 (panel $B$ ) shows that the two factors independently associated with deviation from pre-COVID chemotherapy practice were increasing age (OR between $3 \mathrm{rd}$ and 1st quartiles $3.48,95 \% \mathrm{Cl} 1.71$ to $7.07, \mathrm{p}=<0.01$ ) and palliative intent chemotherapy (OR $3.03,95 \% \mathrm{Cl} 1.28$ to $7.14, \mathrm{p}=0.01$ ), which reflects our modified intent during the lockdown.

\section{Discussion}

When the lockdown was imposed nationwide in India on March 24, the state of West Bengal had 9 confirmed cases, and one death due to COVID19, increasing to 2532 cases and 232 deaths on May 16, 20209. While this reduced casualties from COVID19, lockdown had wide-ranging effects on other healthcare services.

The magnitude of the effect of the COVID 19 induced lockdown on cancer care is emerging globally 10, 11. Data from Prime Minister Jeevandayi Aroyga Yojna (PM-JAY), an universal health insurance scheme, show claims related to oncological care fell by nearly $64 \%$ during the lockdown 12 . Systemic chemotherapy deferrals and delayed start of new patients on chemotherapy were responsible for this decline. Similar reports of disruption in oncological care delivery and its impact have emerged from other healthcare delivery systems in Germany13, Japan 14, Italy15, 16 and the UK17, 18.

To ensure service continuity, services have adopted a system of staff rotations during this crisis 4, 16, 19, 20. Treatment prioritization enabled us to continue delivering safe treatment with reduced staff. Our team was able to reach out to patients with scheduled appointments and provide guidance based on priority levels. This is reflected in a greater drop in follow-up patient visits in breast and prostate cancer patients.

In terms of RT services, we took the decision of not postponing or interrupting RT in patients who were already undergoing treatment. In hindsight this decision was proven correct as to date there is no sign that the epidemic is abating in India despite the lockdown. However, by deferring new starts for priority level 5 patients, we were able to reduce the new starts. This ensured that manpower for planning and treatment could be strategically re-deployed by rotation based $50 \%$ attendance of radiation therapists and medical physicists/dosimetrists to prevent delays and ensure safety in delivering full services for priority level 1-3 and symptomatic level 4 patients. 
For priority level 1-3 patients, this strategy succeeded in implementing more than $90 \%$ of planned starts. Deferments and incomplete treatments were linked primarily to patients being unable to come for treatment. Concurrent chemotherapy was also successfully implemented in the majority. The only factor predicting a change from pre-COVID RT practice was the priority level assigned, which matched with our intent.

The department continued to offer specialized procedures which are highlighted by the fact that one patient successfully underwent Total Body Radiation as a part of the conditioning regimen for bone marrow transplant 21. Additionally, complex planning techniques were used as indicated and no change in planning technique was done. For example, all patients of breast cancer continued to be treated with cardiac sparing using Deep Inspiration Breath Hold 22. This is unlike the experience in some western centers, where similar complex procedures were suspended 23. Significant changes in our dose fractionation schedules were not required as our pre-existing departmental policy was to use hypofractionated radiotherapy wherever it was safe24-26.

The two factors which predicted a deviation from the usual pre-COVID chemotherapy delivery were age and use of palliative chemotherapy - both of which were in line with our proposed departmental protocol (Appendix I). For curative intent patients, on the other hand, a change in dose density was offered. This is reflected in the duration of the deferral of chemotherapy. We also offered G-CSF based prophylaxis to all patients.

Similar experiences from other centers are yet to be reported. No episodes of transmission of COVID 19 from staff to patient or vice versa were observed. Daily pretreatment screening and appropriate counseling of patients and staff may have contributed. Furthermore, with the support of the administration, we were able to ensure that staff could travel from far-flung areas in the city. We must acknowledge the fact that the patients understood the importance of their disease and it's treatment and were motivated to continue on the treatment during this period 27.

The downstream effects of lockdown, in terms of delayed diagnoses or delayed access for newly diagnosed patients in the community, will only become apparent with further follow-up. Modeling results suggest a significant increased risk of death due to delayed treatment and diagnosis in this population28.

\section{Tables}

Table 1: Showing radiotherapy delivery issues for patients planned for RT during the lockdown 


\begin{tabular}{|c|c|c|c|c|c|c|}
\hline Priority Levels & $\stackrel{1}{(N=126)}$ & $2(\mathrm{~N}=18)$ & $3(\mathrm{~N}=14)$ & $\begin{array}{l}4 \\
(\mathrm{~N}=46)\end{array}$ & $\begin{array}{l}5 \\
(\mathrm{~N}=101)\end{array}$ & $\begin{array}{l}\text { Total } \\
(\mathrm{N}=305)\end{array}$ \\
\hline \multicolumn{7}{|c|}{ Radiotherapy Start Status } \\
\hline \multirow[t]{2}{*}{ Started as planned } & 95 & 17 & 11 & 34 & 39 & 196 \\
\hline & $(75.4 \%)$ & $(94.4 \%)$ & $(78.6 \%)$ & $(73.9 \%)$ & $(38.6 \%)$ & $(64.3 \%)$ \\
\hline \multirow[t]{2}{*}{ Deferred Start } & 19 & \multirow[t]{2}{*}{$1(5.6 \%)$} & \multirow{2}{*}{$\begin{array}{l}2 \\
(14.3 \%)\end{array}$} & \multirow{2}{*}{$\begin{array}{l}7 \\
(15.2 \%)\end{array}$} & 37 & \multirow[t]{2}{*}{$66(21.6 \%)$} \\
\hline & $(15.1 \%)$ & & & & $(36.6 \%)$ & \\
\hline \multirow[t]{2}{*}{ Not Started } & \multirow{2}{*}{$\begin{array}{l}12 \\
(9.5 \%)\end{array}$} & \multirow[t]{2}{*}{0} & \multirow{2}{*}{\multicolumn{2}{|c|}{$\begin{array}{l}1(7.1 \%) 5(10.9 \%) \\
(24.8 \%)\end{array}$}} & \multirow[t]{2}{*}{25} & \multirow[t]{2}{*}{$43(14.1 \%)$} \\
\hline & & & & & & \\
\hline \multicolumn{7}{|c|}{ Reason for Not Starting RT $(n=43)$} \\
\hline \multirow[t]{2}{*}{ Unable to come } & 10 & \multirow[t]{2}{*}{0} & \multirow[t]{2}{*}{0} & \multirow{2}{*}{$\begin{array}{l}3 \\
(60.0 \%)\end{array}$} & 20 & \multirow[t]{2}{*}{$33(76.7 \%)$} \\
\hline & $(83.3 \%)$ & & & & $(80.0 \%)$ & \\
\hline \multirow[t]{2}{*}{ Absconded } & \multirow{2}{*}{$\begin{array}{l}2 \\
(16.7 \%)\end{array}$} & \multirow[t]{2}{*}{0} & 1 & 1 & $2(8.0 \%)$ & $6(14.0 \%)$ \\
\hline & & & $(100.0 \%)$ & & & \\
\hline $\begin{array}{l}\text { Physician } \\
\text { Recommended }\end{array}$ & 0 & 0 & 1( & $0.0 \%) 3(1$ & $0 \%)$ & $4(9.3 \%)$ \\
\hline Reason for Start Deferr & $(n=66)$ & & & & & \\
\hline Toxicity & $1(6.2 \%)$ & 0 & 0 & 0 & 0 & $1(1.6 \%)$ \\
\hline Unable to come & 13 & 0 & $6(\varepsilon$ & $5.7 \%)$ & & $50(82.0 \%)$ \\
\hline & $(81.2 \%)$ & & $(100.0 \%)$ & & $82.9 \%)$ & \\
\hline Physician & 2 & 1 & 0 & 1 & 4 & $8(13.1 \%)$ \\
\hline & & $(100.0 \%)$ & & & & \\
\hline Equipment Breakdown & 0 & 0 & 0 & 0 & $2(5.7 \%)$ & $2(3.3 \%)$ \\
\hline
\end{tabular}

Table 2: Showing the patterns of chemotherapy delivery during the period of lockdown. 


\begin{tabular}{|lll|}
\hline \multicolumn{1}{|c|}{ Curative $(n=95)$} & Palliative $(n=124)$ & \\
& & Total $(n=219)$ \\
\hline Site & & \\
\hline
\end{tabular}




\begin{tabular}{|c|c|c|c|}
\hline Breast & $48(50.5 \%)$ & $22(17.7 \%)$ & $70(32.0 \%)$ \\
\hline CNS & $21(22.1 \%)$ & $1(0.8 \%)$ & $22(10.0 \%)$ \\
\hline GI & $5(5.3 \%)$ & $12(9.7 \%)$ & $17(7.8 \%)$ \\
\hline Head Neck & $10(10.5 \%)$ & $15(12.1 \%)$ & $25(11.4 \%)$ \\
\hline Lung & $7(7.4 \%)$ & $58(46.8 \%)$ & $65(29.7 \%)$ \\
\hline Others & $4(4.2 \%)$ & $16(12.9 \%)$ & $20(9.1 \%)$ \\
\hline \multicolumn{4}{|l|}{ ST Deferred } \\
\hline Yes & $31(32.6 \%)$ & 71 (57.3\%) & $102(46.6 \%)$ \\
\hline \multicolumn{4}{|l|}{ Reason Deferred } \\
\hline Toxicity & $9(29.0 \%)$ & $9(12.7 \%)$ & $18(17.6 \%)$ \\
\hline Progression & $1(3.2 \%)$ & $2(2.8 \%)$ & $3(2.9 \%)$ \\
\hline Unable to come & $10(32.3 \%)$ & $18(25.4 \%)$ & $28(27.5 \%)$ \\
\hline Death & 0 & $2(2.8 \%)$ & $2(2.0 \%)$ \\
\hline COVID Related & $3(9.7 \%)$ & $1(1.4 \%)$ & $4(3.9 \%)$ \\
\hline Non COVID Concerns & $8(25.8 \%)$ & $39(60 . \%)$ & $47(46.1 \%)$ \\
\hline \multicolumn{4}{|l|}{ Duration ST Deferred } \\
\hline Median (Range) & $\begin{array}{l}7.500(1.000, \\
72.000)\end{array}$ & $\begin{array}{l}28.000(1.000 \\
60.000)\end{array}$ & $.000(1.000,72.00$ \\
\hline \multicolumn{4}{|l|}{ ST Schedule Changed } \\
\hline Yes & $9(9.5 \%)$ & $9(7.3 \%)$ & $18(8.2 \%)$ \\
\hline \multicolumn{4}{|c|}{ Reason for Change in ST Schedule } \\
\hline COVID Related & $9(100 \%)$ & $2(22.2 \%)$ & $11(61.2 \%)$ \\
\hline Patient Choice & 0 & $1(11.1 \%)$ & $1(5.6 \%)$ \\
\hline Physician Recommendation & 0 & $1(11.1 \%)$ & $1(5.6 \%)$ \\
\hline Progression & 0 & $3(33.3 \%)$ & $3(16.7 \%)$ \\
\hline Toxicity & 0 & $2(22.2 \%)$ & $1.1 \%)$ \\
\hline \multicolumn{4}{|l|}{ Type of Change in ST Schedule } \\
\hline Drug Dose Adjusted & 0 & $2(22.2 \%)$ & $2(11.1 \%)$ \\
\hline Drug Removed & 0 & $1(11.1 \%)$ & $1(5.6 \%)$ \\
\hline
\end{tabular}




\begin{tabular}{|llll|} 
Increased Cycle Duration & $9(100.0 \%)$ & $1(11.1 \%)$ & $10(55.6 \%)$ \\
\hline Regimen Changed & 0 & $5(55.6 \%)$ & $5(27.8 \%)$ \\
\hline
\end{tabular}

\begin{tabular}{|lccc|}
\hline \multicolumn{4}{|l|}{ ST Stopped Completely } \\
\hline Yes & $10(10.5 \%)$ & $18(14.5 \%)$ & $28(12.8 \%)$ \\
\hline Reason for Stopping Systemic Chemotherapy Completely \\
\hline Reason missing & 0 & 3 & 3 \\
\hline Treatment Complete & $1(10.0 \%)$ & 0 & $1(4.0 \%)$ \\
\hline Unable to come & $3(30.0 \%)$ & $4(26.7 \%)$ & $7(28.0 \%)$ \\
\hline Toxicity & $3(30.0 \%)$ & $3(20.0 \%)$ & $6(24.0 \%)$ \\
\hline Progression & $1(10.0 \%)$ & $3(20.0 \%)$ & $4(16.0 \%)$ \\
\hline Non COVID Concerns & $1(10.0 \%)$ & $4(26.7 \%)$ & $5(20.0 \%)$ \\
\hline CoVID Related & $1(10.0 \%)$ & $1(6.7 \%)$ & $2(8.0 \%)$ \\
\hline
\end{tabular}

\section{Declarations}

Acknowledgments: We would like to acknowledge all the members of the radiotherapy department especially, our departmental coordinators Mr Sougata Banerjee and Ms Nandita Das who ensured that treatment delays and deferrals were minimized during this crisis. We would also like to thank all patients who choose to repose their trust in us and continue with the treatment despite facing significant hardships. No extramural or intramural funding was received for this study.

A statement of ethics approval: The study received a waiver from the Institutional Review Board / Ethics committee at our institution as this is an audit (Waiver no: EC/WV/TMC/33/20)

A statement of participant consent: In view of this being an audit without any patient contact or interventions there was a consent waiver from the Ethics Committee.

Competing interests: The authors declare no competing interests.

\section{References}

1. Circulars for Covid-19 | Ministry of Home Affairs | Gol [Internet][cited 2020 Jun 25] Available from: https://www.mha.gov.in/notifications/circulars-covid-19

2. England NHS: Clinical guide for the management of non-coronavirus patients requiring acute treatment: Cancer [Internet]. NHS England , 2020[cited 2020 Jun 15] Available from: 
https://www.england.nhs.uk/coronavirus/wp-content/uploads/sites/52/2020/03/specialty-guid eacute-treatment-cancer-23-march-2020.pdf

3. Pramesh CS, Badwe RA: Cancer Management in India during Covid-19. N Engl J Med 382:e61, 2020

4. Gupta M, Ahuja R, Gupta S, et al: Running of high patient volume radiation oncology department during COVID-19 crisis in India: our institutional strategy [Internet]. Radiat Oncol J , 2020[cited 2020 Jun 25] Available from: https://www.e-roj.org/journal/view.php?doi=10.3857/roj.2020.00199

5. Adhikari SD, Gupta N, Sharma A, et al: Caring of cancer patients during COVID-19: A real-life challenge. Indian J Cancer 57:218-220, 2020

6. Harris PA, Taylor R, Thielke R, et al: Research electronic data capture (REDCap)-a metadata-driven methodology and workflow process for providing translational research informatics support. $\mathrm{J}$ Biomed Inform 42:377-381, 2009

7. Harris PA, Taylor R, Minor BL, et al: The REDCap consortium: Building an international community of software platform partners. J Biomed Inform 95:103208, 2019

8. R Core Team: R: A Language and Environment for Statistical Computing [Internet]. Vienna, Austria, R Foundation for Statistical Computing, 2017Available from: https://www.R-project.org/

9. Welcome to WB HEALTH Portal [Internet][cited 2020 Jun 28] Available from: https://www.wbhealth.gov.in/pages/corona/bulletin

10. Sharpless NE: COVID-19 and cancer. Science 368:1290, 2020

11. De Vincentiis L, Carr RA, Mariani MP, et al: Cancer diagnostic rates during the 2020 "lockdown", due to COVID-19 pandemic, compared with the 2018-2019: an audit study from cellular pathology [Internet]. J Clin Pathol , 2020Available from: http://dx.doi.org/10.1136/jclinpath-2020-206833

12. Smith O, Naib P, Shegal PK, et al: PM-JAY Under Lockdown: Evidence on Utilization PM-JAY Policy Brief, 2020

13. Reuter-Oppermann M, Müller-Polyzou R, Wirtz $\mathrm{H}$, et al: Influence of the pandemic dissemination of COVID-19 on radiotherapy practice: A flash survey in Germany, Austria and PLoS One 15:e0233330, 2020

14. Murakami N, Igaki $\mathrm{H}$, Okamoto $\mathrm{H}$, et al: Preparation for the COVID-19 pandemic in the department of radiation oncology in the National Cancer Center Hospital in Tokyo [Internet]. J Radiat Res , 2020Available from: http://dx.doi.org/10.1093/jrr/rraa031

15. Alterio D, Volpe S, Marvaso G, et al: Head and neck cancer radiotherapy amid COVID-19 pandemic: Report from Milan, Italy [Internet]. Head Neck , 2020Available from: 
http://dx.doi.org/10.1002/hed.26319

16. Krengli M, Ferrara E, Mastroleo F, et al: Running a Radiation Oncology Department at the time of coronavirus: an Italian experience [Internet]. Adv Radiat Oncol , 2020Available from: http://dx.doi.org/10.1016/j.adro.2020.03.003

17. Over 2 million people in backlog for cancer care [Internet]. Cancer Research UK , 2020[cited 2020 Jun 23] Available from: https://www.cancerresearchuk.org/about-us/cancer-news/press-release/202006-01-over-2- million-people-in-backlog-for-cancer-care

18. Lai AG, Pasea L, Banerjee A, et al: Estimating excess mortality in people with cancer and multimorbidity in the COVID-19 emergency. medRxiv 2020.05.27.20083287, 2020

19. Papachristofilou A, Finazzi T, Kohler G, et al: Contingency Plans in a Radiation Oncology Department Amid the 2019-nCoV Outbreak in Switzerland [Internet]. Adv Radiat Oncol , 2020Available from: http://dx.doi.org/10.1016/j.adro.2020.03.012

20. Tey J, Ho S, Choo BA, et al: Navigating the challenges of the COVID-19 outbreak: perspectives from the radiation oncology service in singapore [Internet]. Radiother Oncol , 2020Available from: http://dx.doi.org/10.1016/j.radonc.2020.03.030

21. Achari R, Das A, Mahata A: Total Body Irradiation in Stem Cell Transplant, in Chandy M, Radhakrishnan VS, Sukumaran R (eds): Contemporary Bone Marrow Cham, Springer International Publishing, 2020, pp 1-18

22. Chatterjee $S$, Chakraborty $S$, Moses $A$, et al: Resource requirements and reduction in cardiac mortality from deep inspiration breath hold (DIBH) radiation therapy for left sided breast cancer patients: $A$ prospective service development analysis [Internet]. Pract Radiat Oncol , 2018Available from: http://dx.doi.org/10.1016/j.prro.2018.03.007

23. Beddok A, Calugaru V, Minsat M, et al: Post-lockdown management of oncological priorities and postponed radiation therapy following the COVID-19 pandemic: Experience of the institut curie [Internet]. Radiother Oncol , 2020Available from: http://dx.doi.org/10.1016/j.radonc.2020.05.043

24. Mallick I, Arunsingh M, Chakraborty S, et al: A Phase I/II Study of Stereotactic Hypofractionated Once-weekly Radiation Therapy (SHORT) for Prostate Cancer. Clin Oncol 32:e39-e45, 2020

25. Arunsingh M, Mallick I, Prasath S, et al: Acute toxicity and its dosimetric correlates for high-risk prostate cancer treated with moderately hypofractionated radiotherapy. Med Dosim 42:18-23, 2017

26. Chatterjee S, Arunsingh M, Agrawal S, et al: Outcomes Following a Moderately Hypofractionated Adjuvant Radiation (START B Type) Schedule for Breast Cancer in an Unscreened Non-Caucasian Population. Clin Oncol 28:e165-72, 2016 
27. Ghosh J, Ganguly S, Mondal D, et al: Perspective of Oncology Patients During COVID-19 Pandemic: A Prospective Observational Study From India. JCO Glob Oncol 6:844-851, 2020

28. Sud A, Jones M, Broggio J, et al: Collateral damage: the impact on outcomes from cancer surgery of the COVID-19 pandemic [Internet]. Ann Oncol , 2020Available from: http://dx.doi.org/10.1016/j.annonc.2020.05.009

\section{Figures}

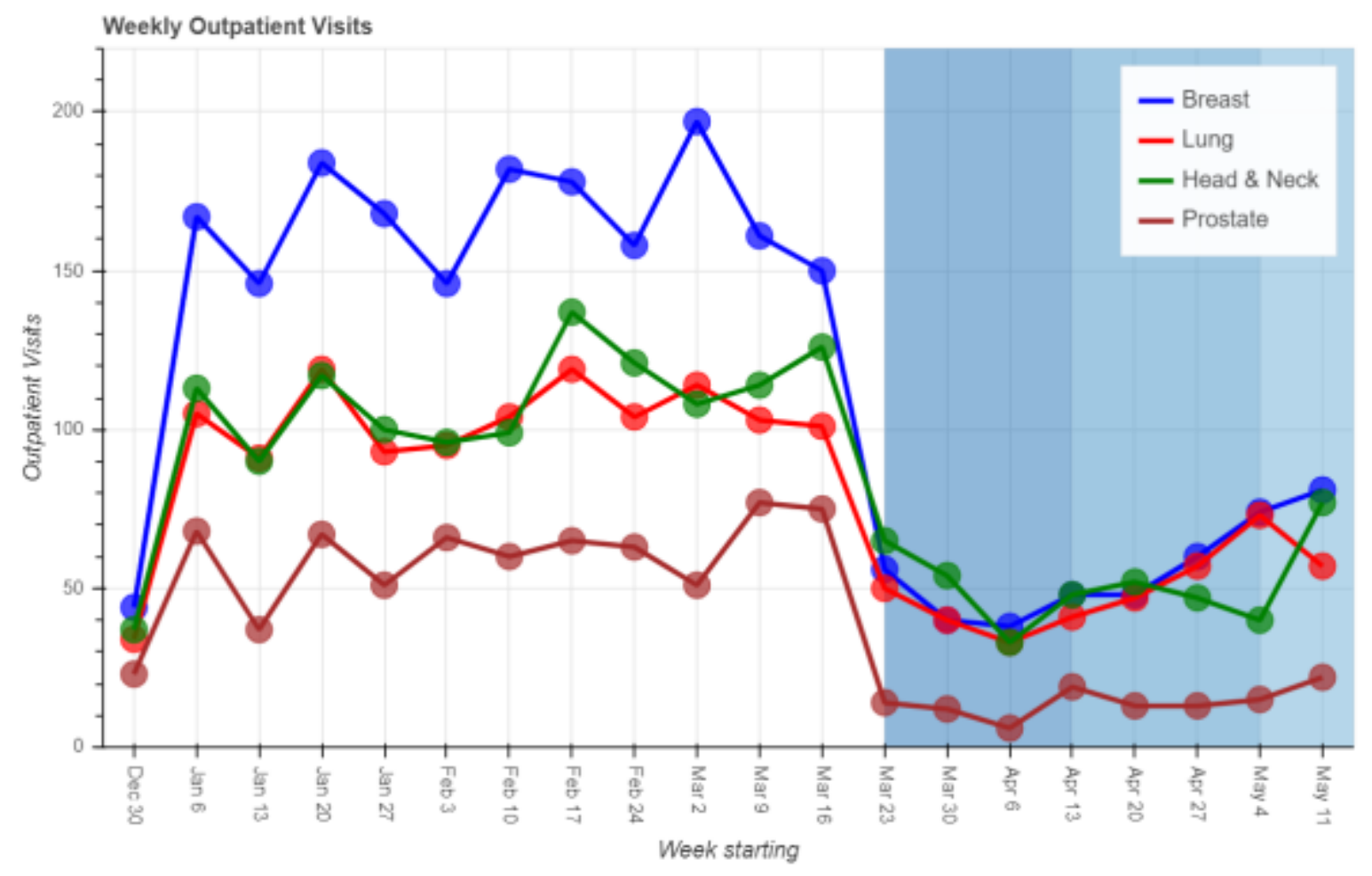

Figure 1

Showing the change in case load in the OPD In the period corresponding to the first, second, and third phases of the lockdown (weeks 13-15, 16-18, and 19-20) for major cancer site groups. 


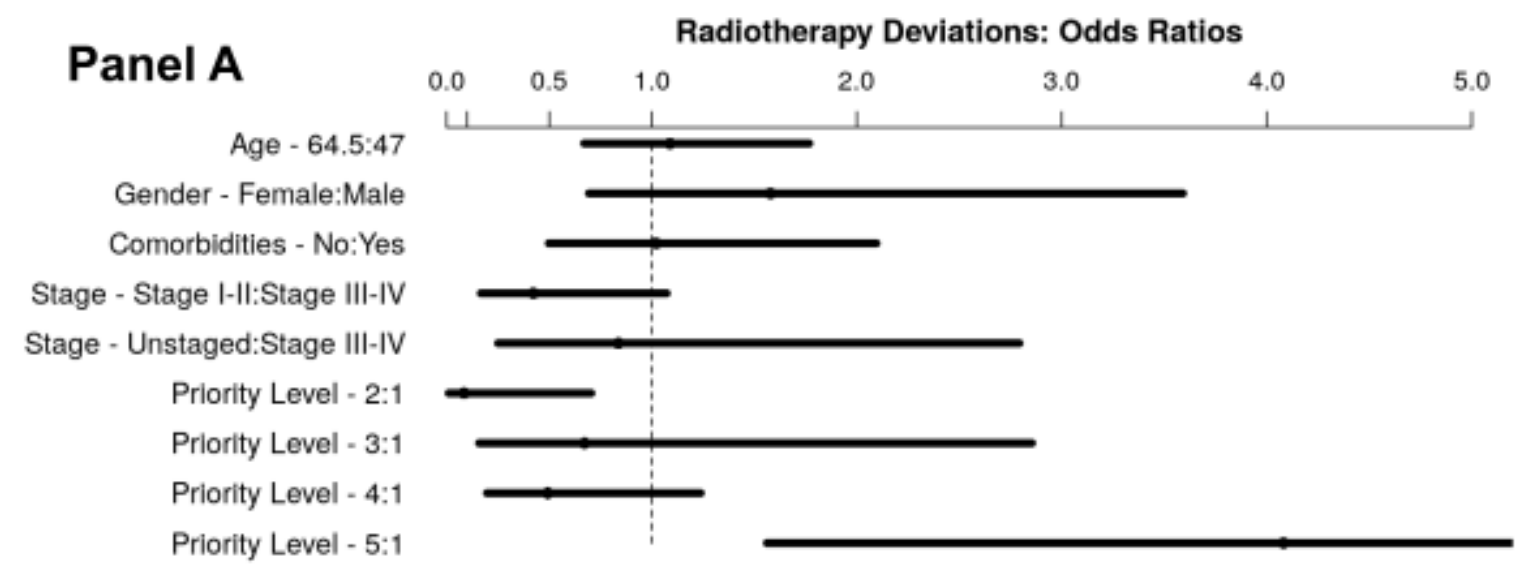

Panel B

\section{Chemotherapy Deviations : Odds Ratios}

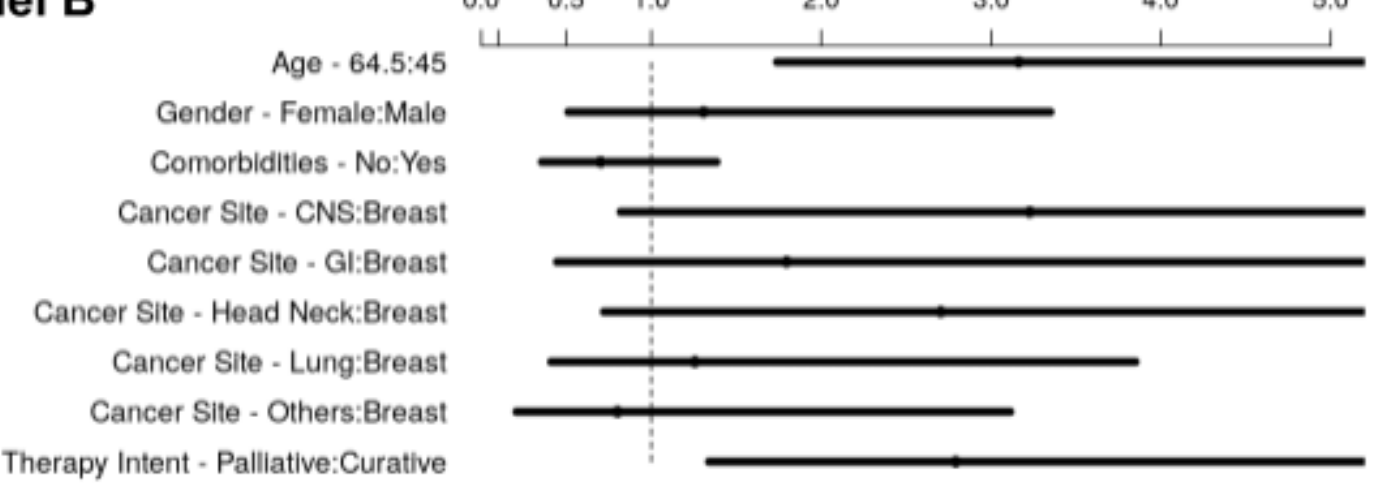

Systemic Therapy Intent - Palliative:Curative

\section{Figure 2}

Showing the Odds Ratio and $95 \%$ confidence intervals of the estimate for each variable obtained from logistic regression for radiotherapy (Panel A) and chemotherapy (Panel B) protocol deviations. The indicator value is toward the right of the colon sign. The $x$-axis of the plot is trimmed at 5.0.

\section{Supplementary Files}

This is a list of supplementary files associated with this preprint. Click to download.

- AppendixIILockdownAudit.pdf

- AppendixILockdownAudit.pdf 